\title{
TIPE1 Suppresses Growth and Metastasis of Ovarian Cancer
}

\author{
Zhenyu Zhang $\mathbb{D}^{1}{ }^{1}$ Minghui Chang $\mathbb{D},{ }^{1}$ Xingguo Song $\left(\mathbb{D},{ }^{1}\right.$ Kangyu Wang $\mathbb{D}{ }^{1}$ \\ Wenjuan Sun $\mathbb{D},{ }^{2}$ Hongxin Ma $\mathbb{D}$, ${ }^{1}$ Xiaohui Yan $\mathbb{D}^{3},{ }^{3}$ Yuhong Sun $\mathbb{D}^{4},{ }^{4}$ Xianrang Song $\mathbb{D},{ }^{1,5}$ \\ and Li Xie $\mathbb{D}^{1}$
}

${ }^{1}$ Department of Clinical Laboratory, Shandong Cancer Hospital and Institute, Shandong First Medical University and Shandong Academy of Medical Sciences, Jinan, Shandong, China ${ }^{2}$ Department of Obstetrics, The Second Hospital of Shandong University, Jinan, Shandong, China

${ }^{3}$ Department of Gynecologic Oncology, Shandong Cancer Hospital and Institute, Shandong First Medical University and Shandong Academy of Medical Sciences, Jinan, Shandong, China ${ }^{4}$ Department of Pathology, Shandong Cancer Hospital and Institute, Shandong First Medical University and Shandong Academy of Medical Sciences, Jinan, Shandong, China ${ }^{5}$ Shandong Provincial Key Laboratory of Radiation Oncology, Shandong Cancer Hospital and Institute, Shandong First Medical University and Shandong Academy of Medical Sciences, Jinan, Shandong, China

Correspondence should be addressed to Li Xie; lxie@sdfmu.edu.cn

Zhenyu Zhang and Minghui Chang contributed equally to this work.

Received 26 January 2021; Accepted 19 May 2021; Published 4 June 2021

Academic Editor: Zhihua Kang

Copyright ( 92021 Zhenyu Zhang et al. This is an open access article distributed under the Creative Commons Attribution License, which permits unrestricted use, distribution, and reproduction in any medium, provided the original work is properly cited.

TIPE1, a newly identified member in TIPE (TNFAIP8) family, plays an important role in tumorigenesis and immune regulation, but its role in ovarian cancer, especially in tumor metastasis, remains unknown. In the current study, we aimed to reveal the protein expression spectrum of TIPE1 in normal human tissues and explored its relationship with metastasis in ovarian cancer. The results of IHC staining showed that TIPE1 protein was not only detected in cytoplasm in most human tissues but also expressed in both cytoplasm and nucleus in squamous epithelium and some epithelial-derived cells with secretory functions, such as esophagus, cervix uteri and ovary, and thyroid gland. Moreover, TIPE1 protein was downregulated in ovarian cancer tissues compared with that in the paracancerous. More importantly, TIPE1 suppressed tumorigenesis and metastasis of ovarian cancer in vitro and in vivo, as evidence shows its ability to suppress growth, colony formation, migration, and epithelial-mesenchymal transition (EMT) of ovarian cancer. Taken together, our results demonstrate the suppressor role of TIPE1 in ovarian cancer metastasis, indicating TIPE1 might be a metastasis predictor and a novel therapeutic target for ovarian cancer.

\section{Introduction}

Ovarian cancer, the most common gynecological malignancy worldwide, has lower incidence than cervical cancer and uterine body cancer; however the death rate is the highest of all gynecological tumors, which seriously poses a threat to women's lives [1]. Despite making unremitting efforts for improving treatment strategies, involving surgery, chemotherapy, and surgery combined with chemotherapy and target therapy in the past forty years, the survival rate had no obvious improvement [2]. Unfortunately, metastasis and recurrence might occur in more than $80 \%$ of patients and the 10 -year rate of disease-free survival among patients is below $15 \%$ [3]. This is largely due to the abnormal growth and increased cell metastasis potential of ovarian cancer cells. Therefore, seeking novel molecular alterations involved in the abnormal cell growth leading to tumor progression would facilitate the realization and development of therapies for ovarian cancer.

TIPE1, a newly identified member of TIPE (tumor necrosis factor- $\alpha$-induced protein 8) family, was first reported to modulate necroptosis and apoptosis in 2008 [4, 5]. Recent studies had suggested that TIPE1 may play a significant role 
in cancer development and progression. Our previous studies found that TIPE1 was downregulated in hepatocellular carcinoma which could induce caspase-mediated apoptosis and inhibit HCC cell growth both in vitro and in vivo [6]. Similar results were confirmed and presented in lung cancer [7], gastric cancer [8], osteosarcoma [9], breast cancer [10, 11], and colorectal cancer [12]. A recent study reported that TIPE1 was decreased in tissue microarrays and inhibited proliferation through inducing apoptosis in ovarian cancer cells [13], but whether TIPE1 related to tumor metastasis was not mentioned. Therefore, it is necessary to explore the relationship between TIPE1 and metastasis and to further clarify its anti-tumor effect in ovarian cancer. In addition, TIPE1 seems to act opposite functions in different cancers. Zhao et al. discovered that TIPE1 could promote cervical cancer progression by repression of p53 acetylation [14] and Liu et al. demonstrated that TIPE1 could accelerate NPC cell proliferation by inhibiting autophagy via the AMPK/mTOR signaling pathway [15]. These findings indicated that the functions of TIPE1 may differ in different tissues. Murine TIPE1 was distributed widely in various mouse tissues [16], but the protein profile of human TIPE1 was still unknown. Hence, it is crucial to clarify the protein expression spectrum of TIPE1 in normal human tissues.

In the current study, we investigated the expression and function of TIPE1 in ovarian cancer. The expression of TIPE in clinical specimens and pathological data indicated that TIPE1 was a suppressor gene in ovarian cancer and it was negatively correlated with cancer metastasis. Furthermore, cell biology assays confirmed that overexpression of TIPE1 could inhibit tumor cell growth and migration. In addition, we presented the expression spectrum of TIPE1 protein in normal human tissues and found that TIPE1 protein was not only detected in cytoplasm of most human tissues, but also detected in nucleus of squamous epithelium and epithelialderived cells, such as esophagus, cervix uteri, ovary, and thyroid gland cells. Our findings revealed the function of TIPE1 in ovarian cancer and suggested that TIPE1 may be a novel therapeutic target for patients.

\section{Materials and Methods}

2.1. Clinical Tissue Samples. The normal human tissue microarray was purchased from the Shanghai Outdo Biotech Company (Shanghai, China). Fresh samples including 18 cancer tissues and 10 non-cancer tissues came from ovarian cancer patients who underwent surgery from March 2018 to July 2018 in Shandong Cancer Hospital and Institute. Informed consent was obtained from all patients before the study was initiated with approval of the Shandong Cancer Hospital and Institute Ethics Committee in accordance with the Declaration of Helsinki. A total of 281 ovarian cancer tissues and 89 non-cancer tissues involved in this study contained the following two parts: 24 ovarian cancer pathological sections and 26 non-cancer pathological sections obtained from the Second Hospital of Shandong University: 5 microarrays containing 257 ovarian cancer tissues and 63 non-cancer tissues purchased and carried out IHC experiments from the Shanghai Outdo Biotech Company (Shanghai, China), Guilin Fanpu Biotech Company
(Guilin, China), and Wuhan Service Biotech Company (Wuhan, China), and all information of ovarian cancer patients was provided by the companies.

2.2. IHC Staining. IHC using the anti-TIPE1 antibody (orb186311, Biorbyt Ltd, Cambridgeshire, UK) was performed according to standard protocols as described previously6. Staining intensity of normal human tissues was scored as follows: no staining $= \pm$; weak staining $=+$; $\bmod$ erate staining $=++$; strong staining $=+++$. To assess the average degree of staining, multiple regions were analyzed, and at least 100 cells were assessed. The histochemistry score of TIPE1 expression in ovarian cancer tissues and noncancer tissues was assessed by computer system [17]. The formula for the $\mathrm{H}$-score is as follows: Histoscore $=\Sigma(\mathrm{I} \times \mathrm{Pi})$, where $\mathrm{I}=$ intensity of staining and $\mathrm{Pi}=$ percentage of stained cells, producing a cytoplasmic score ranging from 0 to 300 , the higher the score, the stronger the positive staining.

2.3. Cell Culture and Transfection. The human ovarian cancer cell lines A2780 and ES2 were purchased from Keygen Biotech (Jiangsu, China). SKOV3 cells were purchased from American Type Culture Collection (ATCC, Manassas, VA, USA). A2780 and ES2 cells were cultured in Dulbecco's modified Eagle's medium (Thermo Fisher, Carlsbad, CA, USA). SKOV3 cells were cultured in McCoy's 5A medium (Thermo Fisher, Carlsbad, CA, USA). All the media were supplemented with $10 \%$ fetal bovine serum (Thermo Fisher) and antibiotics (penicillin/streptomycin, $100 \mathrm{U} / \mathrm{ml}$, Beyotime, Beijing, China) at $37^{\circ} \mathrm{C}$ with $5 \% \mathrm{CO} 2$. The lentivirus-based TIPE1 overexpression system was purchased from Genechem Company (Shanghai, China). The lenti-TIPE1 was employed to infect A2780, ES2 and SKOV3 cells, and puromycin $(2 \mu \mathrm{g} / \mathrm{ml}$, Beyotime, Beijing, China) was added to select the stable TIPE1-expression cells.

2.4. MTT Assay. Cell proliferation analysis was performed by a conventional MTT cell viability assay. Cells were seeded in 96-well plates at density of 2000 per well, and $10 \mu \mathrm{l}$ of the MTT ( $5 \mathrm{mg} / \mathrm{mL}$ in phosphate-buffered saline) was added per well followed by incubation at $37^{\circ} \mathrm{C}$ in $5 \% \mathrm{CO} 2$ for 4 hours before the setting time points. Formazan crystals were solubilized with $100 \mu \mathrm{l}$ of acidified $(0.01 \mathrm{M} \mathrm{HCl}) 10 \%$ sodium dodecyl sulfate (SDS) overnight at $37^{\circ} \mathrm{C}$. Absorbance at $570 \mathrm{~nm}$ was read on a Bio-Rad 680 microplate reader (BioRad Laboratories, CA, USA).

2.5. RNA Extraction and Real-Time RT-PCR. Total RNA was extracted from ovarian cancer cells using Trizol reagent (Invitrogen, Carlsbad, CA, USA) following manufacturer's instructions. After reverse transcription with PrimeScript ${ }^{\mathrm{TM}}$ RT reagent Kit (Takara RR047 A, Tokyo, Japan), the complementary DNA was used for real-time PCR with TB Green ${ }^{\circledR}$ Premix Ex Taq ${ }^{\mathrm{TM}}$ II (Takara RR820 A, Tokyo, Japan). Relative levels of TIPE1 expression were determined with ACTB as the control. The primer for TIPE1 was as follows: forward primer: 5' -CAGTGACCTGCTAGATGAG-3'; reverse primer: 5'”-CAAGGTGCTGAGTGAAGT-3'. 
2.6. Colony Formation Assay. Cells were seeded in 6-well plate at a density of 200 per well and cultured at $37^{\circ} \mathrm{C}$ in $5 \% \mathrm{CO} 2$ for 10-14 days. The plate was then fixed with $4 \%$ paraformaldehyde for 30 minutes and stained with $0.1 \%$ crystal violet for 1 hour. The plate was washed three times with PBS. The numbers of colonies with more than 50 cells were counted.

2.7. Cell Migration Assay. The 24-well plate and matched Transwell chambers (Corning, China) were used for cell migration assay. $1^{*} 105$ cells in $100 \mu \mathrm{l}$ serum-free medium were placed into the upper chamber and $700 \mu \mathrm{l}$ culture medium with $10 \%$ FBS was added to the 24 well plate. After incubating for $48 \mathrm{~h}$ at $37^{\circ} \mathrm{C}$ in $5 \% \mathrm{CO} 2$, the chambers were stained with $0.1 \%$ crystal violet and photographed.

2.8. Wound Healing Assay. Cells were seeded in a 12-well plate at a density of $1{ }^{*} 106$ per well. After growing to $90-95 \%$ confluence in plate and a wound was made by dragging a plastic pipette tip across the cell surface, the wounds were recorded at $37^{\circ} \mathrm{C}$ in $5 \% \mathrm{CO} 2$ for $48 \mathrm{~h}$.

2.9. Western Blot. ES2 and SKOV3 cells were washed twice with PBS and then lysed with Lysis buffer (Beyotime, Shanghai, China) supplemented with Protease Inhibitor (Beyotime, Shanghai, China), and centrifuged at $4^{\circ} \mathrm{C}$ for 15 min. A BCA kit (Thermo Fisher Scientific, Waltham, MA, USA) was used to determine the protein concentration. $40 \mu \mathrm{g}$ quantity of protein was separated on SDS-PAGE and transferred onto PVDF membranes (Millipore, MA, USA). After blocking with $5 \%$ nonfat milk for $2 \mathrm{~h}$, the membrane was incubated with primary antibodies overnight and washed three times for $10 \mathrm{~min}$ with $1 \times$ TBST. Next, the membrane was incubated with the corresponding HRP-conjugated secondary antibodies (anti-mouse or anti-rabbit antibodies, Cell Signaling Technology) for $1 \mathrm{~h}$ at room temperature. The reaction was visualized using Clarity Western ECL substrate (Bio-Rad, CA, USA) or detected by exposure to a film. The antibodies used included E-Cadherin, N-Cadherin, Slug, Snail, cl-Caspase9, cl-Caspase3 from Cell Signaling Technology; Flag, Caspase3, GAPDH from Proteintech Group.

2.10. In Vivo Study. Female BALB/c mice (4-6 weeks of age) were purchased from Lingchang Biotech Company (Shanghai, China). Animals were fed according to protocols approved by the Institutional Animal Care and Use Committee of Shandong Cancer Hospital and Institute with full respect to the EU Directive 2010/63/EU for animal experimentation. Approximately $1 * 107$ Lenti-TIPE1 overexpression SKOV3 cells in $100 \mu \mathrm{l}$ PBS were transplanted subcutaneously into the 10 mice. The lentivirus vector group was used as control. After reaching $100 \mathrm{~mm}^{3}$ in size, size of local tumors and mouse body weight were calculated by measuring two perpendicular diameters (length and width) every three days using a caliper, and the volume was calculated according to the following formula: tumor volume $(\mathrm{mm} 3)=1 / 2 \times$ (length $\times$ square width). Mice were sacrificed after calculating for 19 days and the tumors were isolated and weighed.
2.11. Statistical Analysis. GraphPad Prism 6.02 (GraphPad Software, San Diego, CA, USA) was used for data analysis. Student's $t$-test or one-way ANOVA was applied to determine significant differences between groups. The statistical correlation between clinical characteristics of ovarian cancer and TIPE1 expression levels in tissue was analyzed by the unpaired $t$-test or one-way ANOVA test. $p<0.05$ was considered statistically significant.

\section{Results}

3.1. TIPE1 Protein Expression in Normal Human Tissues. Human TIPE1 protein profile is still unclear till now, so we first detected the protein expression of TIPE1 in normal human tissues, such as digestive system organs (Figure S1), respiratory, muscular and nervous system organs (Figure S2), and reproductive system organs (Figure S3). The IHC results showed that TIPE1 protein was not only detected in the cytoplasm of positively stained cells in most human tissues, but also expressed in both cytoplasm and nucleus of squamous epithelium and some epithelial-derived cells with secretory functions, such as esophagus, cervix uteri, ovary, and thyroid gland. The IHC data are summarized in Table 1. Considering the strongest staining of TIPE1 protein expressed in both cytoplasm and nucleus of ovary cells, we did the following research.

3.2. TIPE1 Was Significantly Decreased in Ovarian Cancer and Negatively Correlated with Tumor Metastasis. To illuminate the function of TIPE1 in both physiologic and pathologic conditions in ovarian cancer, we firstly detected the mRNA expression of TIPE1 in ovarian cancer tissues and non-cancer tissues from patients who underwent surgery from March 2018 to July 2018 in Shandong Cancer Hospital and Institute. The results showed that TIPE1 mRNA was lower in cancer tissues (Figure 1(a)). To confirm the above results, we collected ovarian tissue sections and purchased tissue microarrays including 281 cases of ovarian cancer tissues and 89 cases of normal or benign ovarian tissues. IHC staining level of TIPE1 in these tissues indicated that TIPE1-positive cells were rarely observed among ovarian cancer but obvious in the ovarian stroma (Figure 1(d)). In addition, mean IHC score in cancer tissues was significantly lower than those in non-cancer tissues (Figure 1(b)). More importantly, mean IHC score displayed that protein levels of TIPE1 decreased significantly in ovarian cancer tissues characterized by tumor metastasis (Figures 1(c) and 1(e)). Statistical analysis of clinical characteristics of these patients also demonstrated that TIPE1 expression was negatively correlated with metastasis in ovarian cancer (Table 2). Besides, we had also analyzed the relationship between TIPE1 expression and patient age, tumor size, histology, differentiation grade, and clinical stage of 281 ovarian cancer patients, but unfortunately, no statistical significance between them was observed. 
TABLE 1: TIPE1 protein expression in normal human tissues.

\begin{tabular}{|c|c|c|c|}
\hline \multirow{2}{*}{ Tissues } & \multirow{2}{*}{ Positive cells } & \multicolumn{2}{|c|}{ TIPE1 expression } \\
\hline & & Cytoplasm & Nucleus \\
\hline Esophagus & Stratified squamous epithelium & +++ & ++ \\
\hline \multirow{2}{*}{ Stomach } & Simple columnar epithelium & + & \pm \\
\hline & Parietal cells & ++ & + \\
\hline Duodenum & Simple columnar epithelium & + & \pm \\
\hline Jejunum & Simple columnar epithelium & + & \pm \\
\hline Ileum & Simple columnar epithelium & + & \pm \\
\hline Appendix & Simple columnar epithelium & + & \pm \\
\hline \multirow{2}{*}{ Colon } & Simple columnar epithelium & + & \pm \\
\hline & Plasma cells & ++ & \pm \\
\hline Rectum & Simple columnar epithelium & + & \pm \\
\hline \multirow{2}{*}{ Liver } & Hepatocytes & ++ & \pm \\
\hline & Bile canaliculus & +++ & \pm \\
\hline Pancreas & Islet cells & +++ & \pm \\
\hline Trachea & Pseudostratified ciliated columnar epithelium & ++ & ++ \\
\hline \multirow{2}{*}{ Lung } & Alveolar epithelial cells & + & + \\
\hline & Dust cells & ++ & \pm \\
\hline Myocardium & Myocardial cells & ++ & \pm \\
\hline Artery wall & Smooth muscle cells & \pm & \pm \\
\hline Skeletal muscle & Skeletal muscle cells & ++ & \pm \\
\hline Telencephalon & Neurons & + & + \\
\hline Medulla oblongata & Neurons & + & + \\
\hline Cerebellum & Neurons & \pm & \pm \\
\hline Testis & Spermatogenic epithelium & + & \pm \\
\hline Seminal vesicle & Pseudostratified columnar epithelium & ++ & +++ \\
\hline Prostate & Epithelium & + & \pm \\
\hline Cervix uteri & Stratified squamous epithelium & ++ & ++ \\
\hline Endometrium & Uterine gland & + & + \\
\hline Ovary & Corpus luteum & +++ & +++ \\
\hline Skin & Stratified squamous epithelium & ++ & ++ \\
\hline Urinary bladder & Transitional epithelium & ++ & \pm \\
\hline Spleen & Lymphoid tissue & \pm & \pm \\
\hline Thyroid gland & Follicular epithelial cells & + & + \\
\hline Breast & Acinar cells & ++ & ++ \\
\hline
\end{tabular}

\subsection{TIPE1 Inhibited the Migration of Ovarian Cancer Cells.} To further determine the function of TIPE1 on the biological behaviors of ovarian cancer cells, we employed lentivirus-based TIPE1 expressing system to infect ovarian cancer cell lines, and puromycin was added to select the stable expression cells. Metastasis is an important factor leading to death and recurrence of ovarian cancer. Our previous studies suggested that TIPE1 was associated with ovarian cancer metastasis, so we attempted to evaluate the effect of TIPE1 on migration ability in ovarian cancer cells. As shown in Figure 2(a), western blot assay confirmed that the overexpressed TIPE1 lentivirus had reliable and well efficiency. Secondly, overexpressed TIPE1 could increase the protein level of E-Cadherin but decrease the protein level of N-Cadherin, Slug, and Snail, indicating that overexpressed TIPE1 could inhibit the epithelial-mesenchymal transition of ovarian cancer cells (Figure 2(b)). Furthermore, overexpression of TIPE1 in ES2 and SKOV3 cells decreased the closure of wound area compared to the control, and Transwell assays also confirmed that
TIPE1 down-regulated migratory abilities of ovarian cancer cells (Figure 2(c)).

3.4. TIPE1 Suppressed Ovarian Cancer Cell Proliferation In Vitro. A recent study reported that TIPE1 could inhibit cancer cells by induing apoptosis, and our studies came to the same conclusion. RT-PCR analysis presented the higher expression of TIPE1 mRNA in TIPE1-infected A2780, ES2, and SKOV3 cells (Figure 3(a)). Both MTT assay and colony formation assays were used to estimate the growth abilities of ovarian cancer cells. Overexpression of TIPE1 dramatically decreased cell growth in A2780, ES2 and SKOV3 cells (Figure 3(b)). Colony formation assay also confirmed the inhibiting function of TIPE1 in these cells (Figure 3(c)). Western blot presented that overexpressed TIPE1 increased the expression of cleaved-caspase 3 and cleaved-caspase 9 in ES2 and SKOV3 cells (Figure 3(d)). These results confirmed the inhibition function of TIPE1 in ovarian cancer cell growth and showed a significant anti-tumor activity of TIPE1. 


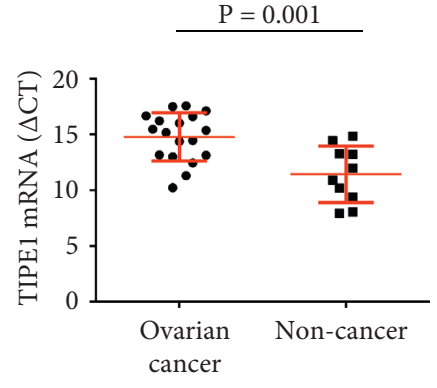

(a)

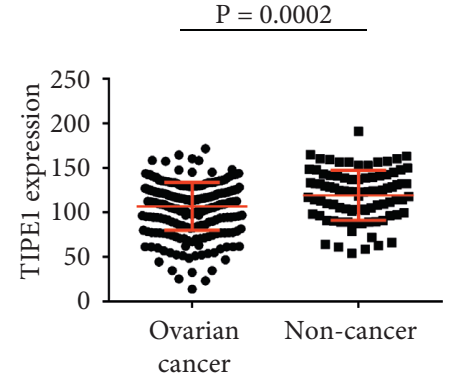

(b)

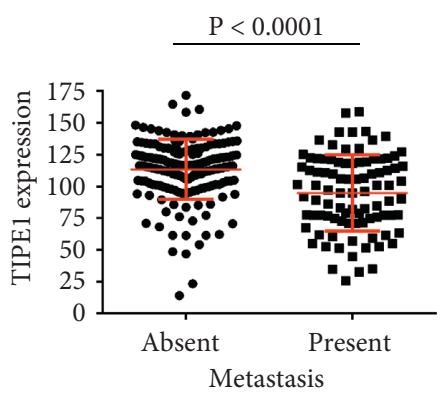

(c)

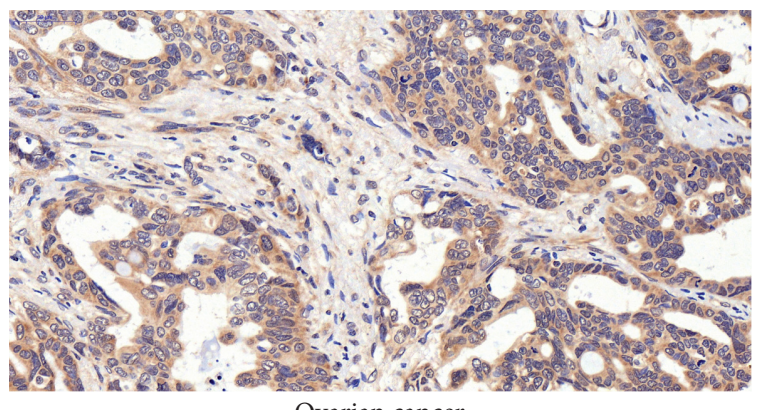

Ovarian cancer

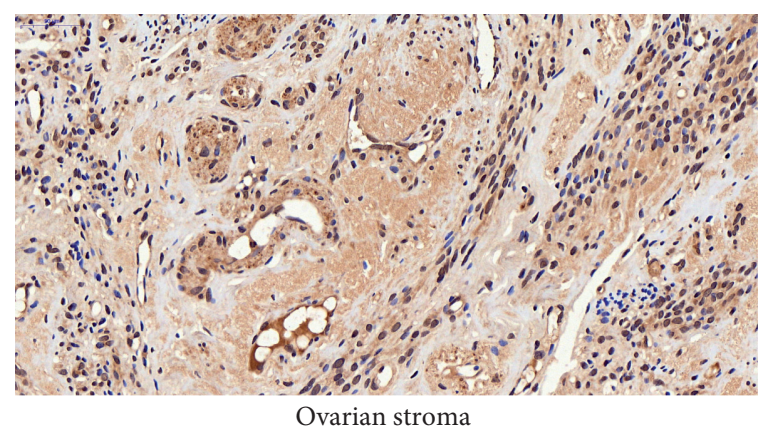

Ovarian stroma

(d)

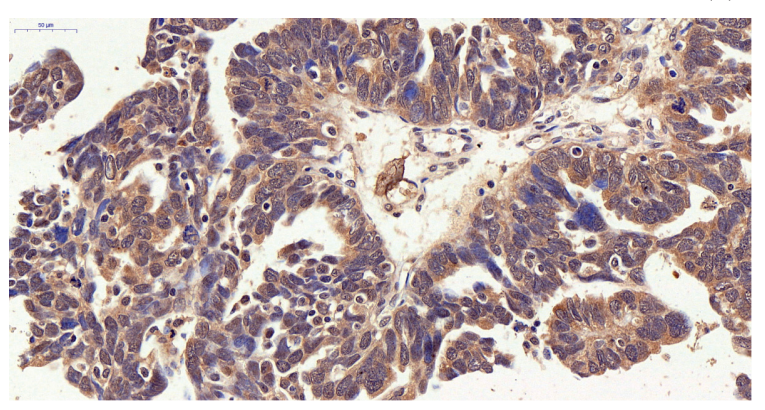

Ovarian cancer with no metastasis

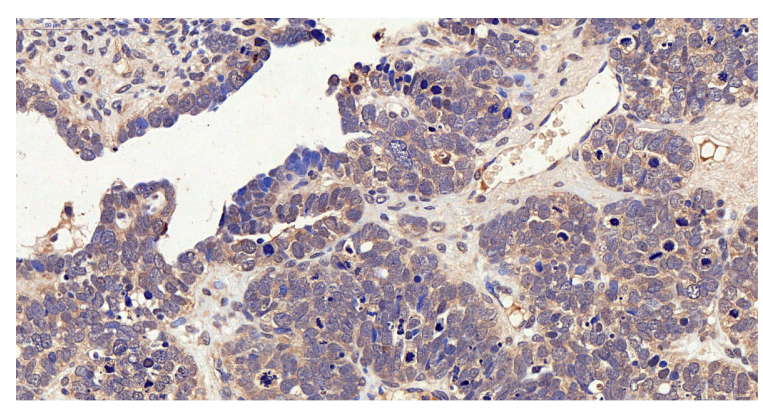

Ovarian cancer with metastasis

(e)

FIGURE 1: TIPE1 was significantly decreased in ovarian cancer and negatively correlated with tumor metastasis. (a) Relative mRNA levels of TIPE1 in ovarian cancer tissues $(n=18)$ and non-cancer tissues $(n=10)$. (b) Immunohistochemistry score analysis of TIPE1 expression in ovarian cancer tissues $(n=281)$ and non-cancer tissues $(n=89)$. (c) Immunohistochemistry score analysis of TIPE1 expression in metastatic ovarian cancer tissues $(n=179)$ and non-metastatic ovarian cancer tissues $(n=102)$. (d) Representative photographs of TIPE1 IHC staining in ovarian cancer tissues and ovarian stroma tissues. Scale bar $=50 \mu \mathrm{m}$. (e) Representative photographs of TIPE1 IHC staining in metastatic ovarian cancer tissues and non-metastatic ovarian cancer tissues. Scale bar $=50 \mu \mathrm{m}$.

3.5. TIPE1 Suppressed Ovarian Cancer Proliferation In Vivo. Furthermore, we investigated whether TIPE1 arrested tumor xenograft growth in vivo. To evaluate the role of TIPE1 in the development of ovarian cancer, we used murine xenograft in Balb/c mice with SKOV3 cells with or without TIPE1 overexpression (Figure 4(a)). Tumor growth curve showed that TIPE1 significantly inhibited the growth of SKOV3 xenograft (Figure 4(b)). Consistently, the average tumor weight of lentivirus-TIPE1 group at the time of killing was noticeably less than that of control group (Figure 4(c)). All these data suggested that deficiency of TIPE1 expression was involved in ovarian cancer cells development.

\section{Discussion}

TIPE family (tumor necrosis factor- $\alpha$-induced protein 8 ) are related to cell death but several members play different roles in tumorigenesis and immune regulation [4, 6, 17-19]. TIPE [20-22] and TIPE3 [18, 23, 24] had been confirmed to possess a carcinogenic effect in tumor development, while TIPE2 $[4,19,25,26]$ was proved to play opposite roles in cell 
TABLE 2: Characteristics of 281 ovarian cancer patients.

\begin{tabular}{|c|c|c|c|}
\hline Clinical characteristics & No. of cases & TIPE1 expression (median \pm SD) & $P$ value \\
\hline $\begin{array}{l}\text { Age }(4 \text { cases } \text { missing }) \\
<60 \text { years } \\
\geq 60 \text { years }\end{array}$ & $\begin{array}{c}203 \\
74\end{array}$ & $\begin{array}{l}112.4 \pm 26.43 \\
111.7 \pm 27.22\end{array}$ & $0.9617^{\mathrm{a}}$ \\
\hline $\begin{array}{l}\text { Tumor size (39 cases missing) } \\
\leq 5 \mathrm{~cm} \\
>5 \mathrm{~cm}\end{array}$ & $\begin{array}{c}55 \\
187 \\
\end{array}$ & $\begin{array}{l}104.3 \pm 30.00 \\
114.2 \pm 26.44\end{array}$ & $0.0833^{a}$ \\
\hline $\begin{array}{l}\text { Histology } \\
\text { Serous } \\
\text { Mucinous } \\
\text { Endometrioid } \\
\text { Clear cell } \\
\text { Squamous } \\
\end{array}$ & $\begin{array}{c}186 \\
52 \\
21 \\
19 \\
3\end{array}$ & $\begin{array}{l}111.5 \pm 27.00 \\
113.5 \pm 27.96 \\
118.0 \pm 26.63 \\
113.5 \pm 23.29 \\
123.8 \pm 19.81 \\
\end{array}$ & $0.5528^{b}$ \\
\hline $\begin{array}{l}\text { Metastasis } \\
\text { Absent } \\
\text { Present }\end{array}$ & $\begin{array}{l}179 \\
102\end{array}$ & $\begin{array}{l}116.1 \pm 24.08 \\
102.2 \pm 28.75\end{array}$ & $<0.0001^{\mathrm{b}}$ \\
\hline $\begin{array}{l}\text { Differentiation grade } \\
\text { Well } \\
\text { Moderate } \\
\text { Poor }\end{array}$ & $\begin{array}{r}42 \\
78 \\
161 \\
\end{array}$ & $\begin{array}{c}115.7 \pm 24.72 \\
110.3 \pm 31.83 \\
112.8 \pm 24.6 \\
\end{array}$ & $0.2968^{b}$ \\
\hline $\begin{array}{l}\text { Clinical stage ( } 73 \text { cases missing) } \\
\text { Stage I } \\
\text { Stage II } \\
\text { Stage III } \\
\text { Stage IV }\end{array}$ & $\begin{array}{c}17 \\
45 \\
109 \\
37\end{array}$ & $\begin{array}{l}109.1 \pm 20.53 \\
112.1 \pm 30.40 \\
112.2 \pm 27.48 \\
115.1 \pm 20.09\end{array}$ & $0.3252^{b}$ \\
\hline
\end{tabular}

${ }^{\mathrm{a}}$ Unpaired $t$-test (two-tailed). ${ }^{\mathrm{b}}$ One-way ANOVA test.

death and tumorigenesis. Unlike the other members, TIPE1 seemed to play more complicated roles in different tumor progressions. We firstly found that TIPE1 was decreased in hepatocelluar carcinoma, increased HCC cell apoptosis, and inhibited cell growth both [6]. Similar results were shown in lung cancer [7], gastric cancer [8], osteosarcoma [9], breast cancer $[10,11]$, and colorectal cancer [12]. However, some researchers discovered that TIPE1 could promote cell proliferation of cervical cancer and nasopharyngeal carcinoma $[15,27]$, suggesting that TIPE1 may have different effects in different types of tumors. This phenomenon might be related to the TIPE1 protein expression in different human tissues. However, the protein spectrum of human TIPE1 remains unclear.

In this study, we discovered that TIPE1 protein was universally expressed in the cytoplasm of most human tissues. However, the expression of TIPE1 protein was also detected in both cytoplasm and nucleus in squamous epithelium of skin, esophagus, cervix uteri, and epithelial-derived cells such as breast and thyroid gland. Interestingly, the TIPE1 expression was barely detected in lymphoid tissue of the spleen (Figure 3(e)), but had stronger expression in the plasma cells (Figure 1(g), red arrows) of colonic tissues than those cells around the intestinal tissues. Similarly, the dust cells expressed higher TIPE1 staining in cytoplasm than those cells surrounding the lung tissues (Figure 2(a), right). As we know, plasma cells are derived from B lymphocytes while dust cells originate from macrophages; both of them are activated immune cells. TIPE1 protein is expressed in activated immune cells but not in non-activated immune cells, suggesting that TIPE1 might play a promotional role in immune system. Among all the mentioned human tissues, it should be noted that ovary had the strongest TIPE1 staining, indicating that the function of TIPE1 in ovary might be typical.

Ovarian cancer is a severe gynecological malignancy and it has the highest mortality of all gynecological tumors [1]. Ovarian cancer does not show any obvious symptoms in its early stages; thus most patients are diagnosed at advanced stages accompanied by multiple metastases, limiting the effectiveness of surgery and chemotherapy [14]. These issues might be explained by the particularity of the tumor microenvironment in ovarian. The tumor microenvironment in ovarian cancer consists of stromal cells and extracellular matrix component, reinforced invasion ability of ovarian cancer cells via the complex cross-talk signaling events $[28,29]$. Therefore, exploring novel genetic sites would be helpful to understand the tumor development and metastasis mechanism of ovarian cancer.

It was reported that TIPE1 was decreased in cancer tissues and inhibited ovarian cancer cell growth [13], but the relationship of TIPE1 and metastasis was not mentioned. In the current study, we proved that TIPE1 was negatively correlated with metastasis and inhibited the migration ability of cells in ovarian cancer. Moreover, overexpressed TIPE1 increased the protein of E-Cadherin but suppressed N-Cadherin, Slug, and Snail expression, which suggested that TIPE1 could inhibit the EMT of ovarian cancer cells. These findings would help us to understand the complex metastatic ovarian tumor associated microenvironment and provide new insights for the development of tumor targeted agents [29]. 


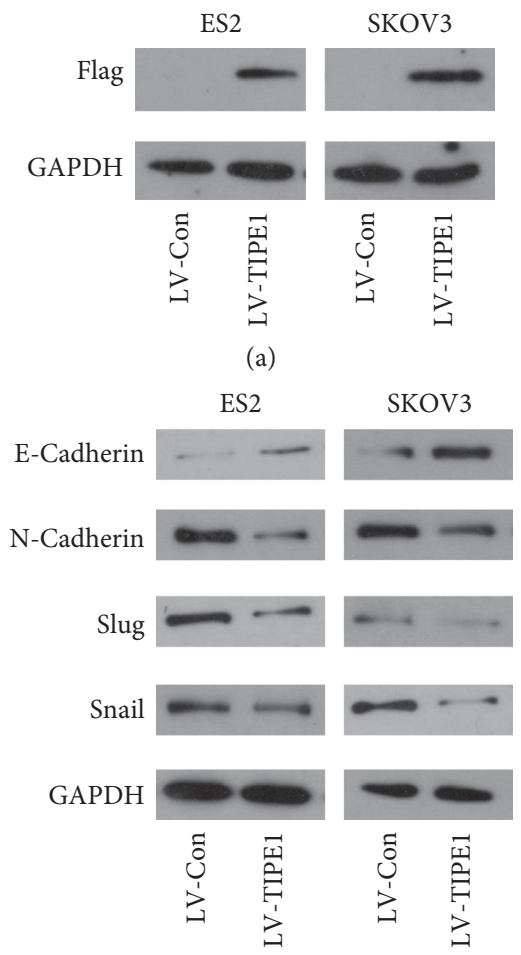

(b)
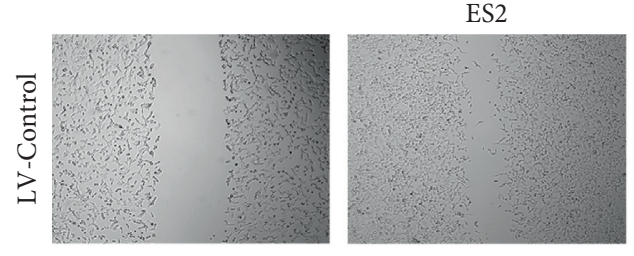

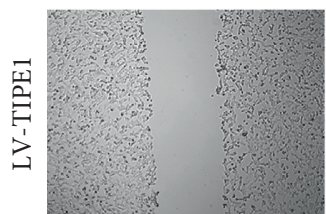

$\mathrm{Oh}$
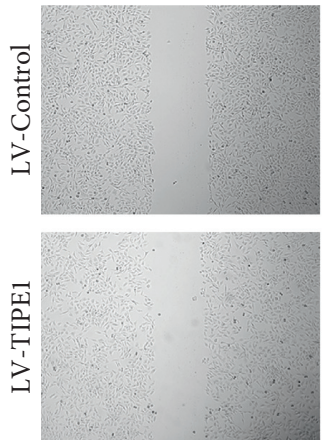

$\mathrm{Oh}$

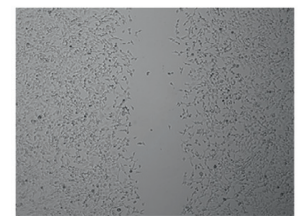

$12 \mathrm{~h}$

SKOV3
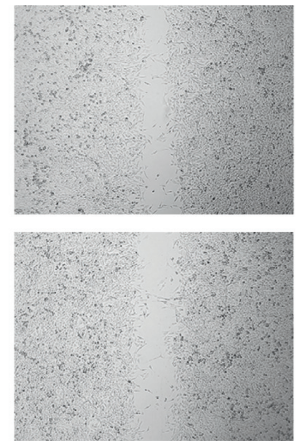

$12 \mathrm{~h}$
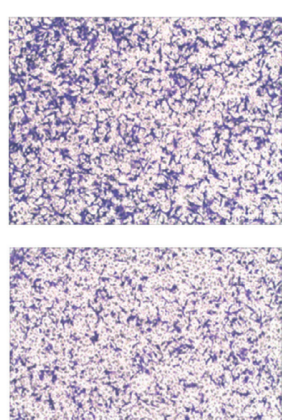

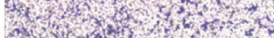
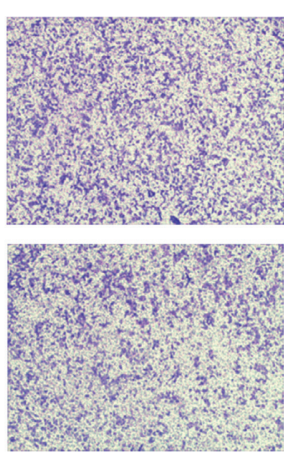

T.

(c)

FIGURE 2: TIPE1 inhibited migration of ovarian cancer cells. (a) The expression of TIPE1 was detected by western blot in ES2 and SKOV3 cells after transfecting lentivirus as indicated. (b) EMT related proteins including E-cadherin, N-cadherin, Slug, and Snail were detected by western blot in ES2 and SKOV3 cells after transfecting lentivirus as indicated. (c) Wound healing assay was performed to determine the migration properties of ES2 and SKOV3 cells. Transwell assay was performed to determine the migration properties of ES2 and SKOV3 cells. Scale bar $=200 \mu \mathrm{m}$. 

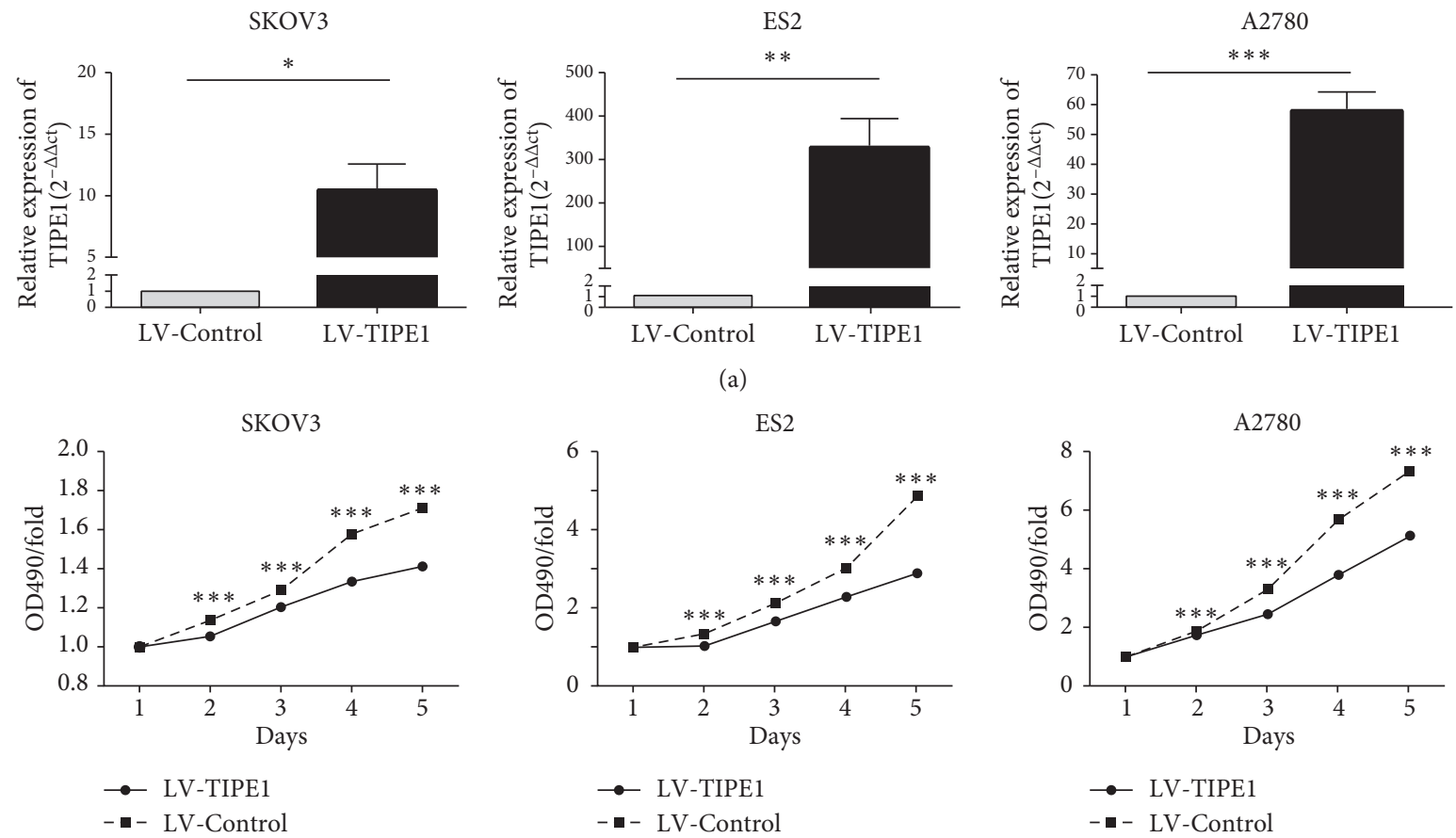

(b)

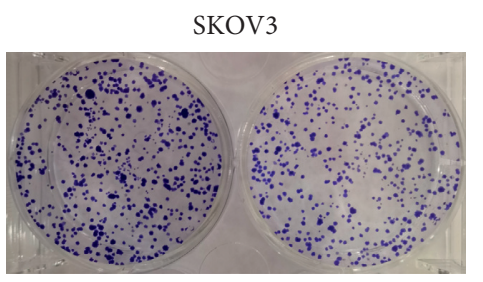

LV-Control

LV-TIPE1

ES2

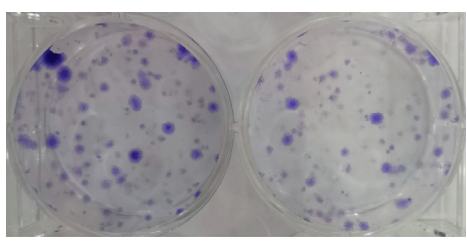

LV-Control

LV-TIPE1

A2780

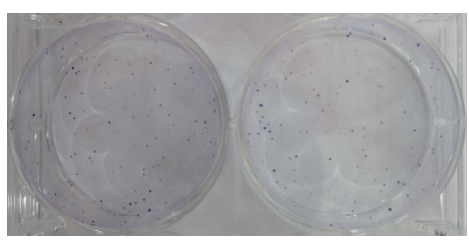

LV-Control

LV-TIPE1
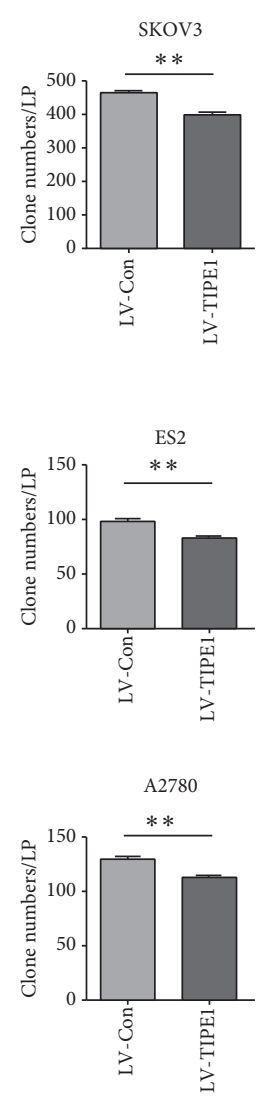

(c)

(d)

FIgURE 3: TIPE1 inhibited growth of ovarian cancer cells in vitro. (a) Real-time PCR was performed to detected mRNA level of TIPE1 after transfecting lentivirus as indicated. (b) Cell growth curve of A2780, ES2, and SKOV3 cells transfected with TIPE1 and control lentivirus. (c) Colony formation analysis of A2780, ES2, and SKOV3 cells transfected with TIPE1 and control lentivirus. (d) Caspase3, cl-Caspase9, and clCaspase 3 were detected by western blot in ES2 and SKOV3 cells after transfecting lentivirus as indicated. The results are presented as mean $\pm \mathrm{SD} ;{ }^{*} p<0.05,{ }^{* *} p<0.01,{ }^{* * *} p<0.001$. 


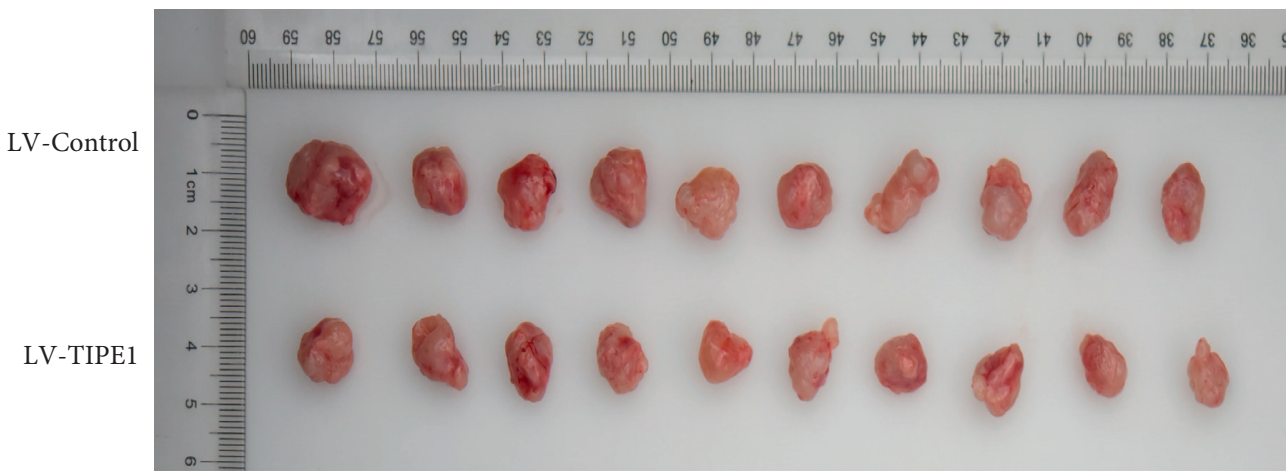

(a)

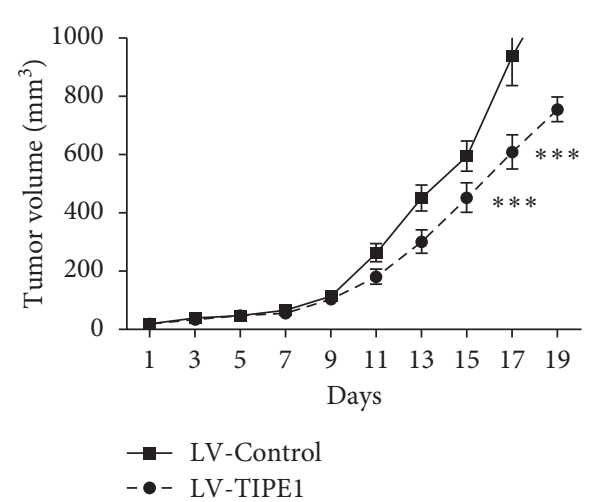

(b)

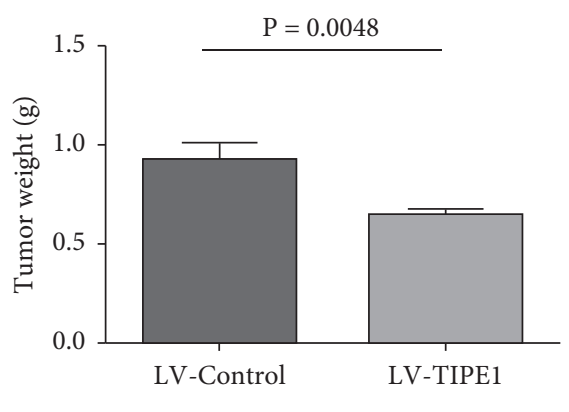

(c)

FIGURE 4: TIPE1 inhibited growth of ovarian cancer in vivo. (a) Photograph of tumors in mice that were injected subcutaneously with SKOV3 cells stably transfected with TIPE1 lentivirus or control lentivirus. (b, c) The average tumor weight and tumor volume in each group over a period of 19 days. The results are presented as mean $\pm \mathrm{SD} ;{ }^{* * *} p<0.001$.

\section{Data Availability}

Data are available upon request to the corresponding author.

\section{Conflicts of Interest}

The authors declare that they have no conflicts of interest.

\section{Authors' Contributions}

Zhenyu Zhang and Minghui Chang contributed equally to this work. L. X. and XR. S. designed experiments; ZY. Z., MH. C., XG. S., KY. W., and HX. M. carried out experiments; WJ. S. and XH. Y. collected ovarian cancer tissues; YH. S. assessed IHC staining; ZY. Z., MH. C., and XG. S. wrote the manuscript and prepared figures. ZY. Z., MH. C., XG. S., KY. W., HX. M., L. X., and XR. S. contributed to analyzing the experimental data. All authors reviewed the manuscript.

\section{Acknowledgments}

This work was supported by grants from the National Natural Science Foundation for Young Scientists of China (81502263 and 81602306), the National Natural Science Foundation of China (81773237), and the Shandong
Provincial Natural Science Foundation of China (BS2015YY019, ZR2019MH004, and ZR2020LZL017).

\section{Supplementary Materials}

Figure S1: TIPE1 protein in digestive system by IHC staining. Figure S2: TIPE1 protein in respiratory system, muscular and nervous system by IHC staining. Figure S3: TIPE1 protein in reproductive system and other tissues by IHC staining. (Supplementary Materials)

\section{References}

[1] F. Bray, J. Ferlay, I. Soerjomataram, R. L. Siegel, L. A. Torre, and A. Jemal, "Global cancer statistics 2018: GLOBOCAN estimates of incidence and mortality worldwide for 36 cancers in 185 countries," CA: A Cancer Journal for Clinicians, vol. 68, no. 6, pp. 394-424, 2018.

[2] J. M. Lee, L. Minasian, and E. C. Kohn, "New strategies in ovarian cancer treatment," Cancer, vol. 125, no. S24, pp. 4623-4629, 2019.

[3] R. L. Dood, Y. Zhao, S. D. Armbruster et al., "Defining survivorship trajectories across patients with solid tumors," JAMA Oncology, vol. 4, no. 11, pp. 1519-1526, 2018.

[4] H. Sun, S. Gong, R. J. Carmody et al., "TIPE2, a negative regulator of innate and adaptive immunity that maintains immune homeostasis," Cell, vol. 133, no. 3, pp. 415-426, 2008. 
[5] J. Hitomi, D. E. Christofferson, A. Ng et al., "Identification of a molecular signaling network that regulates a cellular necrotic cell death pathway," Cell, vol. 135, no. 7, pp. 1311-1323, 2008.

[6] Z. Zhang, X. Liang, L. Gao et al., "TIPE1 induces apoptosis by negatively regulating Racl activation in hepatocellular carcinoma cells," Oncogene, vol. 34, no. 20, pp. 2566-2574, 2015.

[7] X. Wu, Y. Ma, J. Cheng et al., "TIPE1 function as a prognosis predictor and negative regulator of lung cancer," Oncotarget, vol. 8, no. 45, pp. 78496-78506, 2017.

[8] W. Liu, Y. Chen, H. Xie et al., "TIPE1 suppresses invasion and migration through down-regulating $\mathrm{Wnt} / \beta$-catenin pathway in gastric cancer," Journal of Cellular and Molecular Medicine, vol. 22, pp. 1103-1117, 2018.

[9] P. Chen, J. Zhou, J. Li, Q. Zhang, and Q. Zuo, "TIPE1 suppresses osteosarcoma tumor growth by regulating macrophage infiltration," Clinical and Translational Oncology, vol. 21, no. 3, pp. 334-341, 2019.

[10] W. Hu, C.-M. Feng, L.-Y. Liu et al., “TIPE1 inhibits breast cancer proliferation by downregulating ERK phosphorylation and predicts a favorable prognosis," Frontiers in Oncology, vol. 9, p. 400, 2019.

[11] S. Qiu, W. Hu, Q. Ma, Y. Zhao, L. Li, and Y. Ding, “TIPE1 suppresses the invasion and migration of breast cancer cells and inhibits epithelial-to-mesenchymal transition primarily via the ERK signaling pathway," Acta biochimica et biophysica Sinica, vol. 51, no. 10, pp. 1008-1015, 2019.

[12] T. Ye, B. Yang, C. Wang et al., "TIPE1 impairs stemness maintenance in colorectal cancer through directly targeting $\beta$-catenin," Carcinogenesis, vol. 41, pp. 25-35, 2020.

[13] T. Li, L. Jia, Y. Deng, S. Quan, and S. Quan, “TIPE1 impairs ovarian tumor growth by promoting caspase-dependent apoptosis," Oncology Letters, vol. 20, no. 6, p. 1, 2020.

[14] M. Yin, J. Shen, S. Yu et al., "Tumor-associated macrophages (TAMs): a critical activator in ovarian cancer metastasis," OncoTargets and Therapy, vol. 12, pp. 8687-8699, 2019.

[15] Y. Liu, X. Qi, Z. Zhao et al., “TIPE1-mediated autophagy suppression promotes nasopharyngeal carcinoma cell proliferation via the AMPK/mTOR signalling pathway," Journal of Cellular and Molecular Medicine, vol. 24, no. 16, pp. 9135-9144, 2020.

[16] J. Cui, G. Zhang, C. Hao et al., "The expression of TIPE1 in murine tissues and human cell lines," Molecular Immunology, vol. 48, no. 12-13, pp. 1548-1555, 2011.

[17] D. Kumar, P. Gokhale, C. Broustas, D. Chakravarty, I. Ahmad, and U. Kasid, "Expression of SCC-S2, an antiapoptotic molecule, correlates with enhanced proliferation and tumorigenicity of MDA-MB 435 cells," Oncogene, vol. 23, no. 2, pp. 612-616, 2004.

[18] S. A. Fayngerts, J. Wu, C. L. Oxley et al., "TIPE3 is the transfer protein of lipid second messengers that promote cancer," Cancer Cell, vol. 26, no. 4, pp. 465-478, 2014.

[19] Y. Gus-Brautbar, D. Johnson, L. Zhang et al., "The anti-inflammatory TIPE2 is an inhibitor of the oncogenic Ras," Molecular Cell, vol. 45, no. 5, pp. 610-618, 2012.

[20] L. S. Moniz and B. Vanhaesebroeck, "A new TIPE of phosphoinositide regulator in cancer," Cancer Cell, vol. 26, no. 4, pp. 443-444, 2014.

[21] J. M. Lowe, T.-A. Nguyen, S. A. Grimm et al., "The novel p53 target TNFAIP8 variant 2 is increased in cancer and offsets p53-dependent tumor suppression," Cell Death \& Differentiation, vol. 24, no. 1, pp. 181-191, 2017.

[22] Y. Han, Z. Tang, Y. Zhao, Q. Li, and E. Wang, "TNFAIP8 regulates Hippo pathway through interacting with LATS1 to promote cell proliferation and invasion in lung cancer," Molecular Carcinogenesis, vol. 57, no. 2, pp. 159-166, 2018.

[23] K. Lian, C. Ma, C. Hao et al., "TIPE3 protein promotes breast cancer metastasis through activating AKT and NF- $\kappa \mathrm{B}$ signaling pathways," Oncotarget, vol. 8, no. 30, pp. 48889-48904, 2017.

[24] G. Wang, C. Guo, H. Zhao et al., “TIPE3 differentially modulates proliferation and migration of human non-smallcell lung cancer cells via distinct subcellular location," $B M C$ Cancer, vol. 18, no. 1, p. 260, 2018.

[25] Y. Lou, H. Sun, S. Morrissey et al., "Critical roles of TIPE2 protein in murine experimental colitis," The Journal of Immunology, vol. 193, no. 3, pp. 1064-1070, 2014.

[26] S. A. Fayngerts, Z. Wang, A. Zamani et al., "Direction of leukocyte polarization and migration by the phosphoinositide-transfer protein TIPE2," Nature Immunology, vol. 18, no. 12, pp. 1353-1360, 2017.

[27] P. Zhao, X. Pang, J. Jiang et al., “TIPE1 promotes cervical cancer progression by repression of p53 acetylation and is associated with poor cervical cancer outcome," Carcinogenesis, vol. 40, no. 4, pp. 592-599, 2018.

[28] Z. Luo, Q. Wang, W. B. Lau et al., "Tumor microenvironment: the culprit for ovarian cancer metastasis?" Cancer Letters, vol. 377, no. 2, pp. 174-182, 2016.

[29] T.-L. Yeung, C. S. Leung, K.-P. Yip, C. L. Au Yeung, S. T. C. Wong, and S. C. Mok, "Cellular and molecular processes in ovarian cancer metastasis. A review in the theme: cell and molecular processes in cancer metastasis," American Journal of Physiology-Cell Physiology, vol. 309, no. 7, pp. C444-C456, 2015. 